\title{
Damping models in the truncated derivative nonlinear Schrödinger equation
}

\author{
G. Sanchez-Arriaga and J. R. Sanmartin \\ Escuela Tecnica Superior de Ingenieros Aeronauticos, Universidad Politecnica de Madrid, Madrid, Spain \\ S. A. Elaskar \\ CONICET, Departamento de Aeronautica, Universidad Nacional de Cordoba, Cordoba, Argentina
}

(Received 12 April 2007; accepted 11 July 2007; published online 29 August 2007)

\begin{abstract}
Four-dimensional flow in the phase space of three amplitudes of circularly polarized Alfven waves and one relative phase, resulting from a resonant three-wave truncation of the derivative nonlinear Schrödinger equation, has been analyzed; wave 1 is linearly unstable with growth rate $\Gamma$, and waves 2 and 3 are stable with damping $\gamma_{2}$ and $\gamma_{3}$, respectively. The dependence of gross dynamical features on the damping model (as characterized by the relation between damping and wave-vector ratios, $\gamma_{2} / \gamma_{3}, k_{2} / k_{3}$ ), and the polarization of the waves, is discussed; two damping models, Landau $(\gamma \propto k)$ and resistive $\left(\gamma \propto k^{2}\right)$, are studied in depth. Very complex dynamics, such as multiple blue sky catastrophes and chaotic attractors arising from Feigenbaum sequences, and explosive bifurcations involving Intermittency-I chaos, are shown to be associated with the existence and loss of stability of certain fixed point $P$ of the flow. Independently of the damping model, $P$ may only exist for $\Gamma<2\left(\gamma_{2}+\gamma_{3}\right) / 3$, as against flow contraction just requiring $\Gamma<\gamma_{2}+\gamma_{3}$. In the case of right-hand (RH) polarization, point $P$ may exist for all models other than Landau damping; for the resistive model, $P$ may exist for RH polarization only if $\Gamma<\left(\gamma_{2}+\gamma_{3}\right) / 2$. (C) 2007 American Institute of Physics.
\end{abstract}

[DOI: $10.1063 / 1.2768513]$

\section{INTRODUCTION}

Nonlinear Alfven wave interactions are ubiquitous in space plasmas. Strong, nonlinear effects on circularly polarized Alfven waves can be described by the derivative nonlinear Schrödinger (DNLS) equation, ${ }^{1}$ which admits soliton ${ }^{2}$ and traveling wave $\mathrm{e}^{3}$ solutions, and has proved amenable to the inverse scattering method for obtaining general solutions. ${ }^{4}$ Such solitons have been recently observed in the magnetosphere. ${ }^{5}$

The original DNLS equation was a conservative one, but modeling for space applications generally requires including excitation and damping effects. ${ }^{6}$ A DNLS equation modified by damping and excitation has been analyzed in a variety of ways. Extensive numerical simulations have been carried out using spectral analyses. ${ }^{7,8}$ Traveling wave solutions have been analyzed in a driven-DNLS model with diffusive damping $;{ }^{9}$ predicted intermittency-chaos was detected in the solar wind. ${ }^{10}$ Chaotic behavior has been explored by truncating the DNLS equation into three coherently coupled waves near resonance (WRI). ${ }^{8,11,12}$

A space example of an Alfven wave-front involves orbiting conductive tethers, which, if in electrical contact with the ionospheric plasma, radiate charge-carrying Alfven waves that close the current circuit in the ionosphere. ${ }^{13}$ Waves radiated by a tether carrying a steady current form steady trailing structures called Alfven wings. In the distant field, away from the tether, Alfven-wing fronts can be linearly described, and exhibit Airy-function behavior. ${ }^{14}$ Nonlinear effects may be important, however, in the near field. Such nonlinear effects might be affected by the magnetic self-field generated by the very current of the tether, ${ }^{15}$ in a suggested tether experiment, growth rate could be attained by modulating the current in the tether, and thus the background magnetic field. ${ }^{11}$ This would excite an Alfven wave at frequency one-half the modulation frequency through certain parametric instability (magnetoacoustic pumping). ${ }^{16,17}$

Here we discuss how damping models affect solutions of the DNLS equation. We use a truncation of the equation to represent three modes under resonant interaction (WRI), wave 1 being linearly unstable and waves 2 and 3 damped. ${ }^{11}$ This results in a four-dimensional (4D) flow of three wave amplitudes and one relative phase. For waves 2 and 3 equally damped, left-hand (LH) polarized Alfven waves exhibited a hard transition to complex dynamics in a 3D phase-space: no matter how small the growth rate of wave 1 , a chaotic attractor could fully develop. ${ }^{11}$ No such transition was found for right-hand $(\mathrm{RH})$ polarization, paralleling the known fact that only LH time-harmonic solutions of the DNLS equation are modulationally unstable. ${ }^{18}$

Here we explore a wide range in growth rate of wave 1 so as to ascertain whether gross features in dynamical behavior are dependent on the damping model (a WRI approximation possibly failing on a number of conditions it requires), and how broad is the domain in parameter space exhibiting complex dynamic behavior, depending on whether waves are LH- or RH-polarized. We explicitly consider both resistive and linear Landau damping, for which the damping ratio for waves 2 and 3 is simply related to the respective wavenumber ratio, thus reducing the dimension of parameter space.

The fully three-wave truncation model of the DNLS 
equation is discussed in the next section; stationary points of the flow are discussed in Sec. III. Phase-space dynamics is analyzed in Secs. IV and V under Landau and resistive damping, respectively. Conclusions are discussed and summarized in Sec. VI.

\section{DNLS THREE-MODE TRUNCATION}

The DNLS equation describes the evolution of circularly polarized Alfven waves of finite amplitude propagating parallel or near-parallel to an unperturbed uniform magnetic field in a cold, homogeneous plasma, using a two-fluid, quasineutral approximation with electron inertia and current displacement neglected. Taking the unperturbed magnetic field $B_{0}$ in the $z$ direction, the DNLS equation reads ${ }^{1-3}$

$$
\frac{\partial \phi}{\partial t}+\frac{\partial}{\partial z}\left[\phi\left(1+|\phi|^{2}\right)\right] \pm \frac{i}{2} \frac{\partial^{2} \phi}{\partial z^{2}}+\hat{\gamma} \phi=0
$$

$\hat{\gamma}$ being some damping/growth operator. As usual, we introduced dimensionless time and position $t \Omega_{c i} \rightarrow t, z \Omega_{c i} / V_{A}$ $\rightarrow z$, using ion cyclotron frequency and Alfven velocity, and a perturbed complex-2D magnetic field $\phi \equiv\left(B_{x} \pm i B_{y}\right) / 2 B_{0}$. The upper (lower) sign corresponds to LH (RH) -polarized Alfven waves.

We look for solutions in the form

$$
\phi \approx \sum_{j=1}^{3} a_{j}(t) e^{i\left[k_{j} z-\omega_{j} t+\psi_{j}(t)\right]}
$$

involving three modes under wave number resonance $2 k_{1}$ $=k_{2}+k_{3}$, with mode 1 excited and modes 2 and 3 damped. We can then derive equations for amplitudes $a_{1}, a_{2}, a_{3}$, and relative phase

$$
\beta \equiv \pi+\nu t+\psi_{1}+\psi_{2}+\psi_{3},
$$

with frequency mismatch ${ }^{11}$

$$
\nu \equiv 2 \omega_{1}-\omega_{2}-\omega_{3}= \pm \frac{\omega_{1}^{2}}{\Omega_{c i}}\left(\frac{k_{3}-k_{2}}{k_{3}+k_{2}}\right)^{2},
$$

which arises from the wave dispersion relation,

$$
\omega_{j}=V_{A} k_{j}\left(1 \mp \frac{1}{2} \frac{V_{A} k_{j}}{\Omega_{c i}}\right) \Rightarrow \omega_{j}=k_{j} \mp \frac{1}{2} k_{j}^{2} .
$$

The mismatch is positive (negative) for LH (RH) polarization. The divergence of the flow in phase-space may be shown to be constant,

$$
\begin{gathered}
\frac{\partial}{\partial a_{1}^{2}} \frac{d a_{1}^{2}}{d t}+\frac{\partial}{\partial a_{2}^{2}} \frac{d a_{2}^{2}}{d t}+\frac{\partial}{\partial a_{3}^{2}} \frac{d a_{3}^{2}}{d t}+\frac{\partial}{\partial \beta} \frac{d \beta}{d t} \\
=-2\left(\gamma_{2}+\gamma_{3}-\Gamma\right),
\end{gathered}
$$

with $\gamma_{2}, \gamma_{3}$ damping rates of mode 2,3 , and $\Gamma \equiv-\gamma_{1}>0$ growth rate of mode 1 .

Introducing normalized amplitudes and time,

$$
\begin{aligned}
& \left(\gamma_{2}+\gamma_{3}\right) t \rightarrow t, \\
& a_{1}^{2} \rightarrow a_{1}^{2}\left(\gamma_{2}+\gamma_{3}\right) / \sqrt{k_{2} k_{3}},
\end{aligned}
$$

$$
\begin{aligned}
& a_{2}^{2} \rightarrow \frac{a_{2}^{2}\left(\gamma_{2}+\gamma_{3}\right)}{k_{2}+k_{3}} \sqrt{\frac{k_{2}}{k_{3}}}, \\
& a_{3}^{2} \rightarrow \frac{a_{3}^{2}\left(\gamma_{2}+\gamma_{3}\right)}{k_{2}+k_{3}} \sqrt{\frac{k_{3}}{k_{2}}},
\end{aligned}
$$

the system of equations, with $a_{3}$ replaced by the amplitude ratio $r \equiv a_{3} / a_{2}$, reads

$$
\begin{aligned}
& \dot{a}_{1}= \hat{\Gamma} a_{1}-r a_{1} a_{2}^{2} \sin \beta, \\
& \dot{a}_{2}=-\frac{\delta}{1+\delta} a_{2}+r a_{1}^{2} a_{2} \sin \beta, \\
& \dot{r}=-\frac{1-\delta}{1+\delta} r+\left(1-r^{2}\right) a_{1}^{2} \sin \beta, \\
& \dot{\beta}=\hat{\nu}-2 a_{1}^{2}\left(\bar{V}-\frac{1+r^{2}}{2 r} \cos \beta\right)-2 r a_{2}^{2} \cos \beta \\
&+\frac{a_{2}^{2}}{2 \bar{V}}\left(\kappa+\frac{r^{2}}{\kappa}\right) .
\end{aligned}
$$

Equations (6a)-(6d) involve four control parameters

$$
\begin{aligned}
& \kappa \equiv \frac{k_{2}}{k_{3}}<1\left(\bar{V} \equiv \frac{1+\kappa}{2 \sqrt{\kappa}}>1\right), \\
& \delta \equiv \frac{\gamma_{2}}{\gamma_{3}}<1, \\
& \hat{\Gamma} \equiv \frac{\Gamma}{\gamma_{2}+\gamma_{3}}<1, \\
& \hat{\nu} \equiv \frac{\nu}{\gamma_{2}+\gamma_{3}} .
\end{aligned}
$$

We took $\gamma_{3}>\gamma_{2}$ and assumed that damping increases with wave number; also, we considered negative flow divergence in Eq. (4). The number of parameters is reduced when using particular damping models, such as Landau $(\gamma \propto k)$ and resistive $\left(\gamma \propto k^{2}\right)$ models, ${ }^{11}$

$\gamma=\frac{m_{e}}{m_{i}} \sqrt{\frac{\pi k_{B} T_{e}}{2 m_{e} V_{A}^{2}}} \exp \left(-\frac{m_{e} V_{A}^{2}}{2 k_{B} T_{e}}\right) \times \frac{\theta^{2}}{4} \times V_{A} k \rightarrow \delta=\kappa$,

$\gamma \approx \frac{V_{A}^{2} k^{2}}{2 \Omega_{c i} \Omega_{c e} \tau} \rightarrow \delta=\kappa^{2}$

\section{EXISTENCE OF STATIONARY POINTS}

As we shall see in Secs. IV and V, the existence and stability of certain stationary or fixed point severely affects the dynamics of system (6a)-(6d). Fixed points of the system describe Alfven waves for which nonlinear and dispersive effects balance each other to keep amplitudes and relative phase constant, and are obtained by requiring the right-hand side of Eqs. (6a)-(6d) to vanish. Phase-space coordinates of possible fixed points would then be given as 


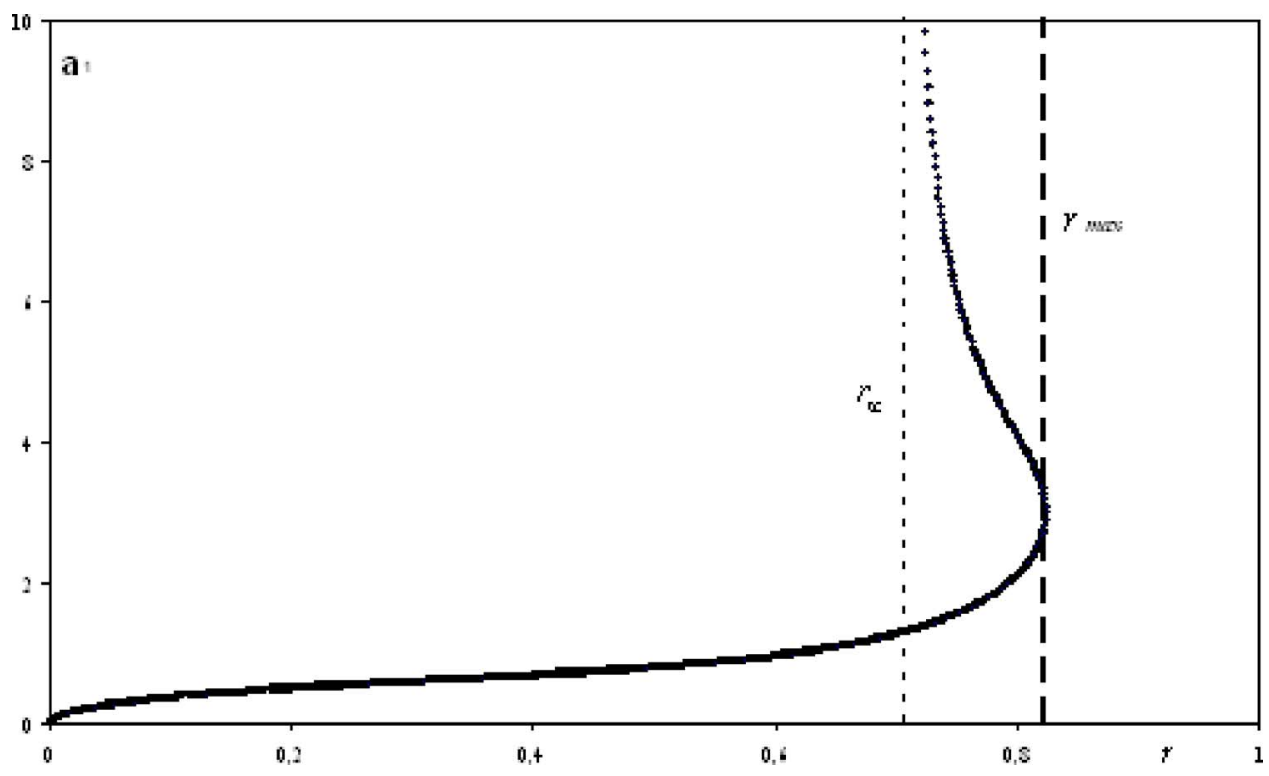

FIG. 1. Characteristic projection on plane $r-a_{1}$ of the fixed-points line of system (6a)-(6d) in the surface $a_{2}=0$, at $\hat{\Gamma}=0$.

$$
\begin{aligned}
& r=\sqrt{\delta}, \\
& \hat{\nu} \sin \beta=F(\kappa, \delta, \hat{\Gamma})-(1-2 \hat{\Gamma}) \cos \beta, \\
& (1+\delta) a_{1}^{2} / \sqrt{\delta}=a_{2}^{2} \sqrt{\delta} / \hat{\Gamma}, \\
& =1 / \sin \beta,
\end{aligned}
$$

with

$$
F \equiv \frac{1+\kappa}{1+\delta} \sqrt{\frac{\delta}{\kappa}}-\frac{\delta+\kappa^{2}}{(1+\kappa) \sqrt{\kappa \delta}} \hat{\Gamma} .
$$

It is convenient to rewrite Eq. (9b) as

$$
\cos \beta=\frac{F(1-2 \hat{\Gamma}) \pm \hat{\nu} \sqrt{\hat{\nu}^{2}+(1-2 \hat{\Gamma})^{2}-F^{2}}}{\hat{\nu}^{2}+(1-2 \hat{\Gamma})^{2}} .
$$

There may thus exist two fixed points $P$ and $P^{*}$, one, or none. We will call $P\left(P^{*}\right)$ the point with the upper (lower) sign in Eq. $\left(9 b^{\prime}\right)$.

A clearly necessary condition for the existence of two fixed points,

$$
F^{2}<\hat{\nu}^{2}+(1-2 \hat{\Gamma})^{2},
$$

is not a sufficient condition, however. Setting $\sin \beta$ positive as required by Eqs. (9c) and (9d) further narrows the parametric domain of existence. Full conditions for the existence of point $P$ with $F \hat{\nu}>0$ can be shown to be

$$
\begin{aligned}
& F^{2}<\hat{\nu}^{2}+(1-2 \hat{\Gamma})^{2} \text { for } \hat{\Gamma}<1 / 2, \\
& (1-2 \hat{\Gamma})^{2}<F^{2}<\hat{\nu}^{2}+(1-2 \hat{\Gamma})^{2} \text { for } 1 / 2<\hat{\Gamma},
\end{aligned}
$$

whereas for $F \hat{\nu}$ negative we find

$$
F^{2}<(1-2 \hat{\Gamma})^{2} \text { for } \hat{\Gamma}<1 / 2,
$$

no fixed point $P$ for $1 / 2<\hat{\Gamma}$.

Conditions for point $P^{*}$ to exist are obtained by just changing ranges $\hat{\Gamma}<1 / 2$ and $1 / 2<\hat{\Gamma}$ above. Clearly, the existence of fixed points may depend on the polarity of the waves (LH having $\hat{\nu}>0$, RH having $\hat{\nu}<0$.)

Some simple results can be readily obtained. First, we prove that point $P^{*}$ is always unstable. The characteristic equation for both fixed points takes the form

$$
\lambda^{4}+2(1-\hat{\Gamma}) \lambda^{3}+C \lambda^{2}+D \lambda+E=0
$$

where coefficients $C$ and $D$ are intricate functions of parameters but $E$ just reads

$$
E=\frac{8 \hat{\Gamma} \delta}{(1+\delta)^{2}} \frac{1-2 \hat{\Gamma}-F \cos \beta}{\sin ^{2} \beta}
$$

A fourth-degree equation with two coefficients having opposite sign has a root with positive real part, corresponding to instability. Using $\sin \beta>0$ and Eq. (9b) [and $\left.\left(9 b^{\prime}\right)\right]$ we find

$$
E=\frac{\mp 8 \hat{\Gamma} \delta \sqrt{\hat{\nu}^{2}+(1-2 \hat{\Gamma})^{2}-F^{2}}}{(1+\delta)^{2} \sin \beta}
$$

which is clearly negative for $P^{*}$.

Next, general results on the existence of fixed points can be derived for arbitrary damping laws in the limit $\hat{\Gamma} \rightarrow 0^{+}$, which, as already noticed, was discussed in Ref. 11 with reference to a hard transition to chaos. In the plane $a_{2}=0$, which is then an invariant surface of system (6a)-(6d), there is a full line of fixed points $\beta(r), r\left(a_{1}\right)$, 


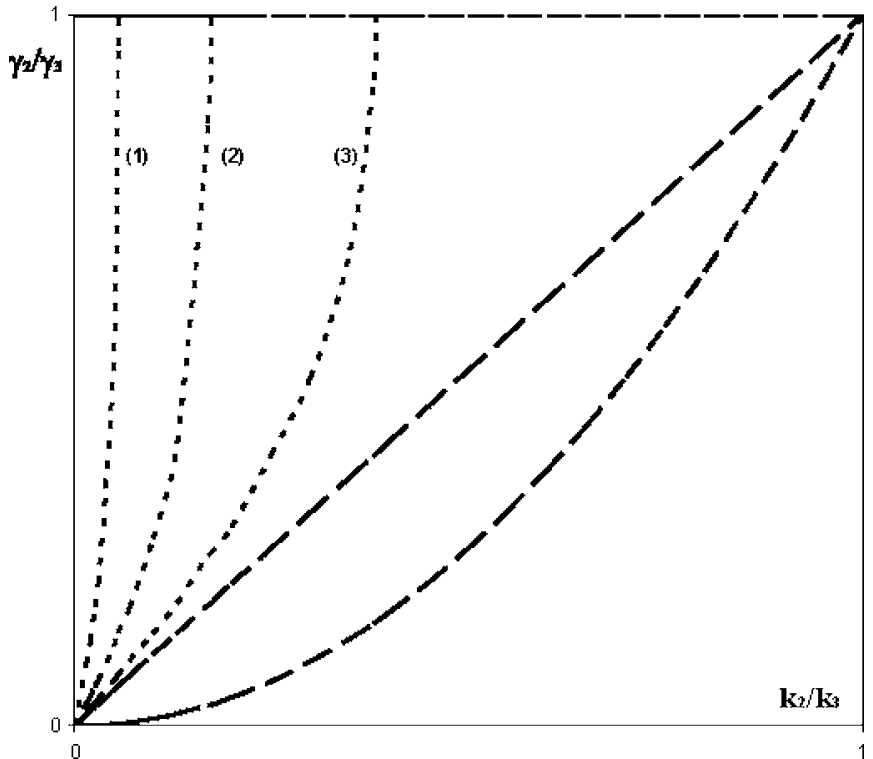

FIG. 2. Landau $(\delta=\kappa)$, resistive $\left(\delta=\kappa^{2}\right)$, and equal $(\delta=1)$ damping laws; coordinate axes are wave vector $(\kappa)$ and damping $(\delta)$ ratios for modes 2 and 3. Also shown is the line family in Eq. (19) applying in the $\hat{\Gamma}=0$ case, with $\hat{\nu} \equiv \nu /\left(\gamma_{3}+\gamma_{2}\right)=2,1$, and 0.5 for lines (3), (2), and (1), respectively; for each line there are no fixed points to its left, both $P$ and $P^{*}$ between line and diagonal, and just $P$ below the diagonal.

$$
\begin{aligned}
& \frac{1-\delta}{1+\delta} r=\left(1-r^{2}\right) a_{1}^{2} \sin \beta, \\
& \hat{\nu}=2 a_{1}^{2}\left(\bar{V}-\frac{1+r^{2}}{2 r} \cos \beta\right) .
\end{aligned}
$$

As $a_{1}$ increases along this line from 0 to $\infty, r$ increases from 0 to $r_{\infty}=\sqrt{ }$, first going through a maximum $r_{\max }$, however, with $a_{1}(r)$ double-valued between $r_{\infty}$ and $r_{\max }$ (Fig. 1). The relative values of $r_{\infty}, r_{\max }$, and $r_{P}=r_{P^{*}}=\sqrt{ } \delta$, as given by (9a) independently of $\hat{\Gamma}$, determine the very existence of $P$ and $P^{*}$ in the limit $\hat{\Gamma} \rightarrow 0^{+}$.

Figure 2 depicts the damping laws $\delta=1$ (leading to a 3D flow with $r=1$ that was discussed in Ref. 11), $\delta=\kappa$, and $\delta=\kappa^{2}$, in the square $0<\kappa<1,0<\delta<1$, covering all laws. For $\hat{\Gamma}=0$, Eq. (9b) becomes

$$
\begin{aligned}
& \hat{\nu} \sin \beta=F_{0}-\cos \beta, \\
& F_{0}=\frac{1+\kappa}{1+\delta} \sqrt{\frac{\delta}{\kappa}}>0 .
\end{aligned}
$$

In the lower right half of Fig. $2, \delta<\kappa$ (which includes the resistive model), we have $F_{0}<1$; Eq. (18a) may be satisfied for either sign of $\hat{\nu}$, that is, we may have either polarization. Also, since we now have $\sqrt{ } \delta<r_{\infty}$, there is just one fixed point; consideration of conditions (12a) and (13a) for $P$, and the corresponding conditions (12b) and (13b) for $P^{*}$, shows that $P$ exists, whereas $P^{*}$ does not.

In the upper right half, $\delta>\kappa$, we have $F_{0}>1$ in Eq. (18a), only LH polarization $(\hat{\nu}>0)$ being thus possible. Since we also have $\sqrt{ } \delta>r_{\infty}$, conditions (12a) and (12b), which then apply, show that both points $P$ and $P^{*}$ do exist for
$F_{0}^{2}<1+\hat{\nu}^{2}$, corresponding to $\sqrt{ } \delta<r_{\max }$, with $a_{1 P}<a_{1}\left(r_{\max }\right)$ $<a_{1 P^{*}}$ in Fig. 1, whereas neither point exists for $F_{0}^{2}>1+\hat{\nu}^{2}$ (with $\sqrt{ } \delta>r_{\max }$ ). Also drawn in Fig. 2 is the separatrix line

$$
F_{0} \equiv \frac{1+\kappa}{1+\delta} \sqrt{\frac{\delta}{\kappa}}=\sqrt{1+\hat{\nu}^{2}}
$$

for a few $\hat{\nu}$ values, with the region to the left of each line corresponding to no fixed point. As $\hat{\nu} \rightarrow 0$, the line in Eq. (19) collapses into the diagonal $\left(r_{\max } \rightarrow r_{\infty}\right)$. At the diagonal (Landau model), we have $\sqrt{ } \delta=r_{\infty}, F_{0}=1$ with $a_{1 P^{*}=\infty}$.

\section{LANDAU DAMPING MODEL}

In this section, we consider the damping/wave-vector relation $\delta=\kappa$, at the diagonal in Fig. 2, as exemplified by Landau damping. Equation $(9 b)$ now reads

$$
\hat{\nu} \sin \beta=1-\hat{\Gamma}-(1-2 \hat{\Gamma}) \cos \beta .
$$

Discussing the existence of fixed points is here particularly simple because Eq. (20) is independent of $\kappa$ (or $\delta$ ). Further, for the excitation regime considered,

$$
0<\hat{\Gamma}<1
$$

we have

$$
\begin{aligned}
& F_{L} \equiv 1-\hat{\Gamma}>1-2 \hat{\Gamma}, \\
& F_{L}>0 .
\end{aligned}
$$

It follows that there is now no point $P$ in the case of $\mathrm{RH}$ polarization $(\hat{v}<0)$, for which condition (13a) applies, showing that $P$ might only exist for $F_{L}^{2}<(1-2 \hat{\Gamma})^{2}$ with $1-2 \hat{\Gamma}$ $>0$, which is a condition opposite to Eq. (22a). As we shall see in the next section, this is a singular property of Landau damping, flows with other damping regimes in Fig. 2 allowing for fixed point $P$ under RH polarization.

For LH polarization, condition (12a) here becomes

$$
\hat{\nu}>\sqrt{\hat{\Gamma}(2-3 \hat{\Gamma})}, \quad \hat{\Gamma}<1 / 2 .
$$

In turn, condition $(12 b)$ reads

$$
\hat{\nu}>\sqrt{\hat{\Gamma}(2-3 \hat{\Gamma})}, \quad 1 / 2<\hat{\Gamma}<2 / 3 .
$$

The domain of existence of point $P$ in the $\hat{\Gamma}-\hat{\nu}$ plane, as follows from Eqs. (23a) and (23b), is shown in Fig. 3, where we also show the domain of existence of $P^{*}$. We have $\beta_{P}$ $\rightarrow \pi, a_{1 P} \rightarrow \infty$ as $\hat{\Gamma} \rightarrow 2 / 3$, and $\beta_{P^{*}} \rightarrow 0, a_{1 P^{*}} \rightarrow \infty$ as $\hat{\Gamma} \rightarrow 0$, thus recovering the result at the end of the preceding section.

The stability of $P$ does involve the third parameter, $\kappa$. For $\hat{\Gamma} \rightarrow 0^{+}$, the characteristic equation for the fixed point has a root reading $\lambda \approx i \alpha_{0} \sqrt{\hat{\Gamma}}+\alpha_{1} \hat{\Gamma}$. The two-branch curve for marginal stability $\alpha_{1}(\kappa, \hat{\nu})=0$ is represented in Fig. 4 (full lines), where it determines two bounded domains with $P$ unstable. Figure 4 also shows the corresponding domains of instability for $\hat{\Gamma}=0.1$ (dashed lines); note that now point $P$ only exists for $\hat{\nu}>\sqrt{0.1 \times(2-0.3)}=\sqrt{0.17}$, as given by condition (23a) and represented in Fig. 3. 


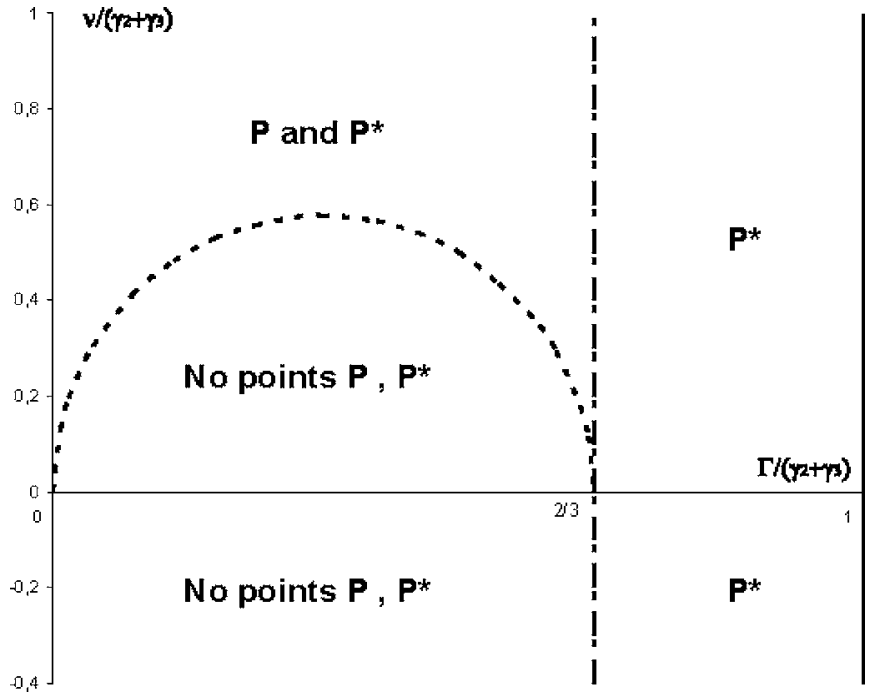

FIG. 3. Domains of existence of points $P$ and $P^{*}$ in the Landau damping model. Coordinate axes are normalized growth rate $\hat{\Gamma}$ of mode 1 and frequency mismatch $\hat{v}$; the lower bound for $P$ is given by Eqs. (23a) and (23b).

Figures 5-7 show attractor-bifurcation diagrams for $a_{1 \max }$ (maximum $a_{1}$ in an attractor) versus $\hat{\nu}$, for $\hat{\Gamma}=0.1$ and three values of $\kappa$ within the domain, to the right, where $P$ is unstable in Fig. 4. In all cases, point $P$ is a stable attractor except for the $\hat{v}$ range within that unstable domain for the corresponding $\kappa$ value. For $\hat{v}$ decreasing, $P$ goes through a bifurcation at a return point with $\hat{\nu}=\sqrt{ } 0.17$, where it becomes point $P^{*}$, which we had shown to be always unstable.

In Fig. 5, a stable periodic orbit is born through a supercritical Hopf bifurcation when point $P$ reaches the $\hat{v}$ value at either boundary of the (right) unstable domain in Fig. 4 for $\kappa=0.7$. In Fig. 6 , for $\kappa=0.9$, the periodic orbit born at the Hopf bifurcations becomes unstable at two $\hat{\nu}$ values through cyclic folds (saddle-node bifurcations), where the larger Floquet multiplier reaches +1 . Note that, for a limited $\hat{\nu}$ range just below 0.9 , there are five attractors: two unstable fixed points, one unstable periodic orbit, and two stable periodic orbits.

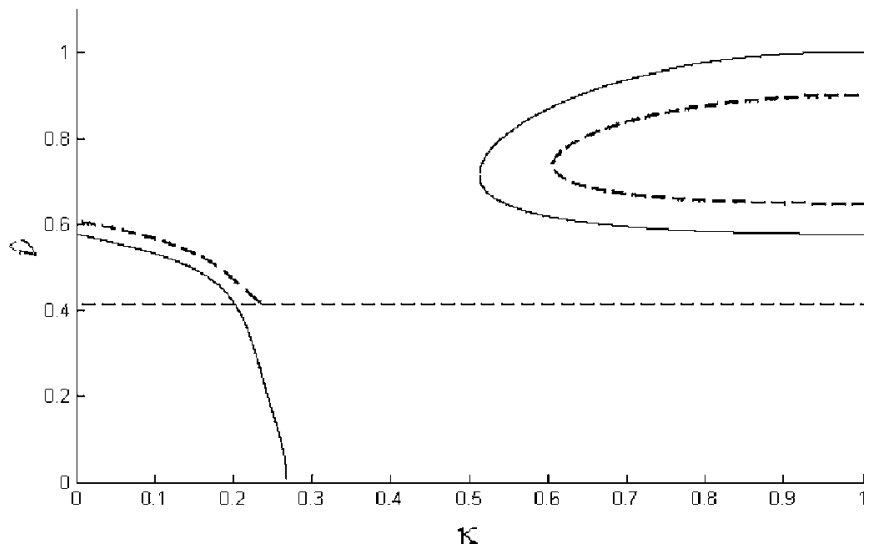

FIG. 4. Stability of point $P$ in the Landau model. $P$ is unstable within the bounded domains to the left (low $\kappa$ ) and right (high $\kappa$ ) for $\hat{\Gamma} \rightarrow 0^{+}$(full lines) and $\hat{\Gamma}=0.1$ (dashed lines). For $\hat{\Gamma}=0.1$, there is no point $P$ for $\hat{\nu}$ below $\downarrow 0.17$, as following from Eq. (23a).

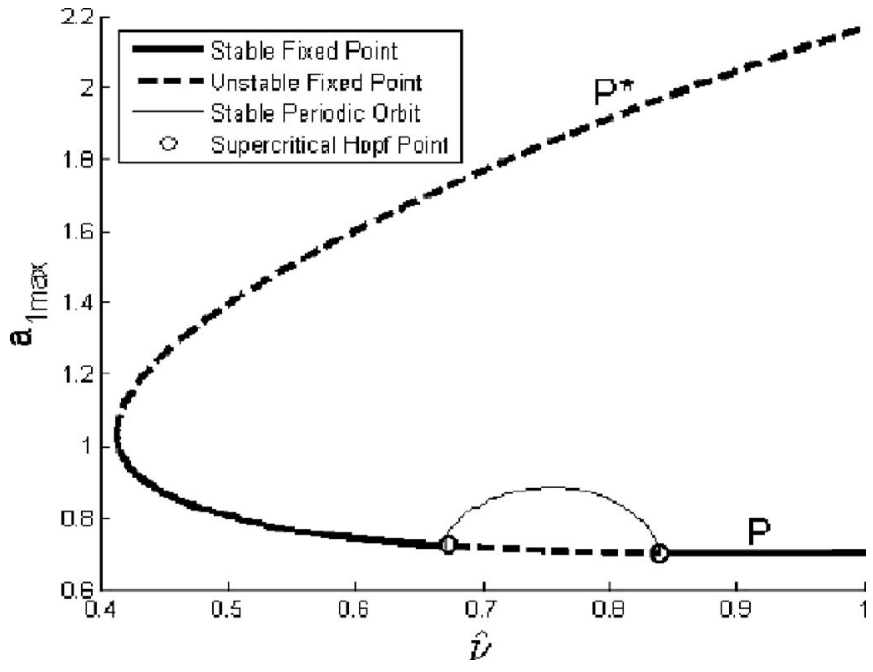

FIG. 5. Attractor-bifurcation diagram at $\hat{\Gamma}=0.1$ and $\kappa=0.7$ in the Landau model

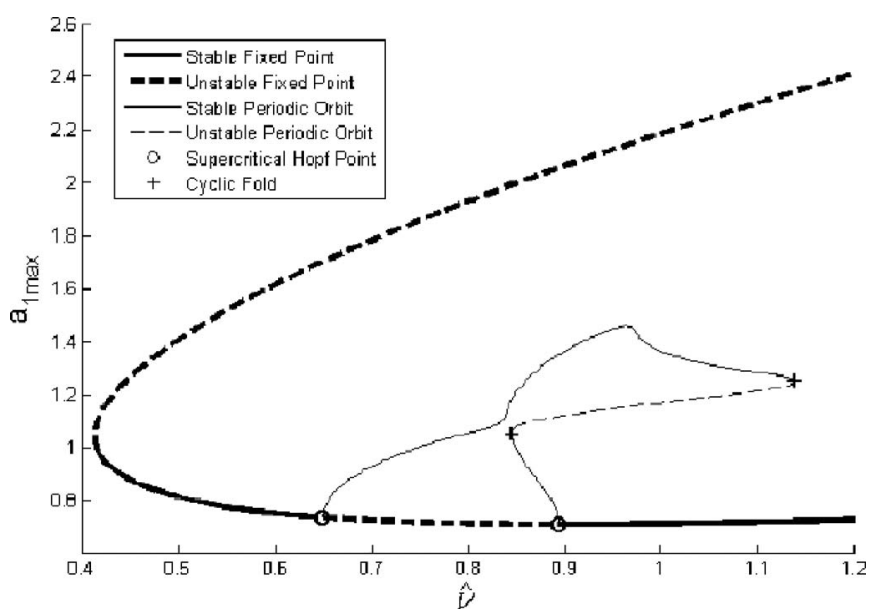

FIG. 6. Same as in Fig. 5 at $\kappa=0.9$.

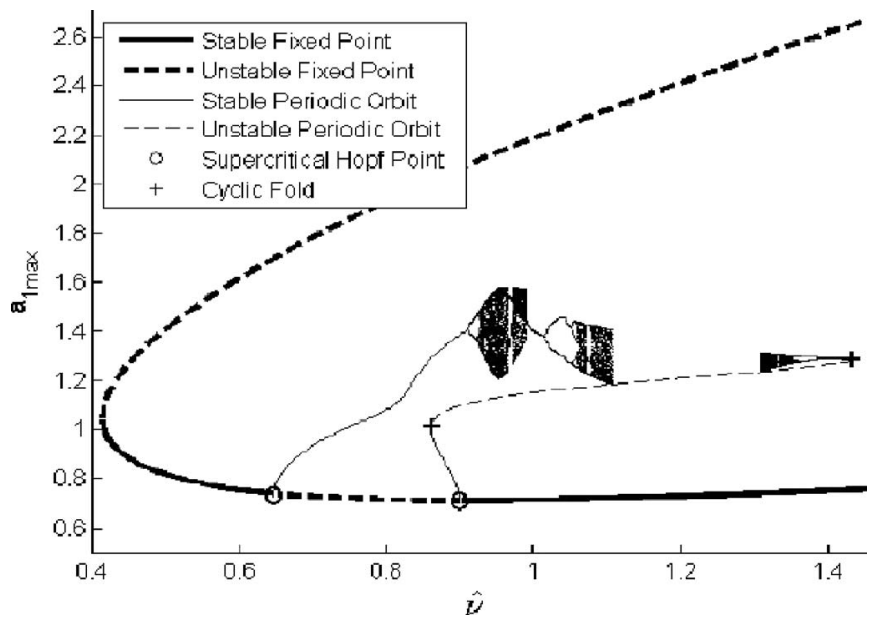

FIG. 7. Same as in Fig. 5 at $\kappa=0.95$. 


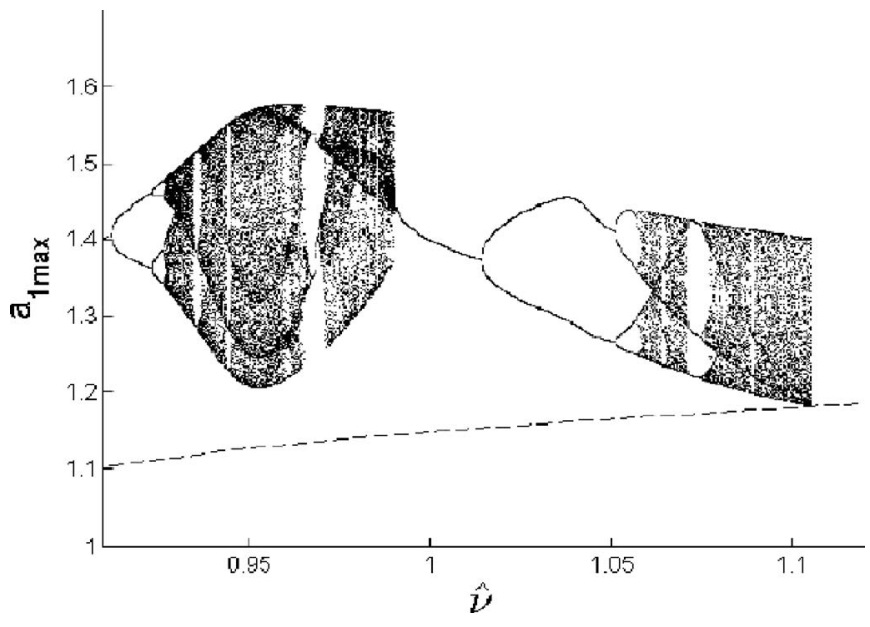

FIG. 8. Zoom of the $0.9-1.1 \hat{\nu}$ range in Fig. 7 .

The bifurcation diagram for $\kappa=0.95$ is extremely rich (Fig. 7). The periodic orbit born through a Hopf bifurcation at $\hat{\nu}$ about 0.63 becomes unstable when its smaller Floquet multiplier reaches -1 , with $a_{1 \max } \approx 1.4$. The orbit of a double period then born goes through the same period-doubling bifurcation; a Feigenbaum sequence follows, ending in a chaotic attractor that extends up to about $\hat{\nu}=0.99$ (Fig. 8). To its right in the figure there is a periodic orbit, which, for $\hat{\nu}$ back decreasing, loses stability with $a+1$ Floquet multiplier when reaching the chaotic attractor. This is a Shilnikov-like explosive bifurcation, with the periodic attractor exploding into the much larger chaotic one. Within this last attractor at $\hat{\nu}<0.99$, the phase trajectory spends long times in the phasespace proximity of what will be the periodic orbit for $\hat{\nu}>0.99$, but these laminar stages are followed by irregular bursts away from that phase region, in what appears as intermittency of type I.

For increasing $\hat{\nu}$, the above periodic orbit starts a new period-doubling sequence ending in a second chaotic attractor. This attractor collapses in a boundary crisis or "blue sky" catastrophe (with the flow approaching point $P$, which is now stable) when it collides, past $\hat{\nu} \approx 1.1$, with an unstable periodic orbit born at $\hat{\nu}$ about 0.85 through a cyclic fold of a periodic orbit (itself born at the supercritical Hopf bifurcation of point $P$ at $\hat{v}$ about 0.9 ). The unstable periodic orbit ends through another cyclic fold at $\hat{\nu} \approx 1.43$ in a new periodic orbit, which gives rise at decreasing $\hat{v}$ to a third Feigenbaum sequence and a third chaotic attractor. There is a second blue sky catastrophe at $\hat{\nu}$ about 1.3, with the flow again approaching point $P$.

We have computed the Lyapunov exponents for the chaotic attractor at $\hat{\nu}=0.95$ in Fig. $8(\kappa=0.95, \hat{\Gamma}=0.1)$, which are

$$
\begin{aligned}
& \lambda_{1} \approx 0.03, \\
& \lambda_{2} \approx 0, \\
& \lambda_{3} \approx-0.86,
\end{aligned}
$$

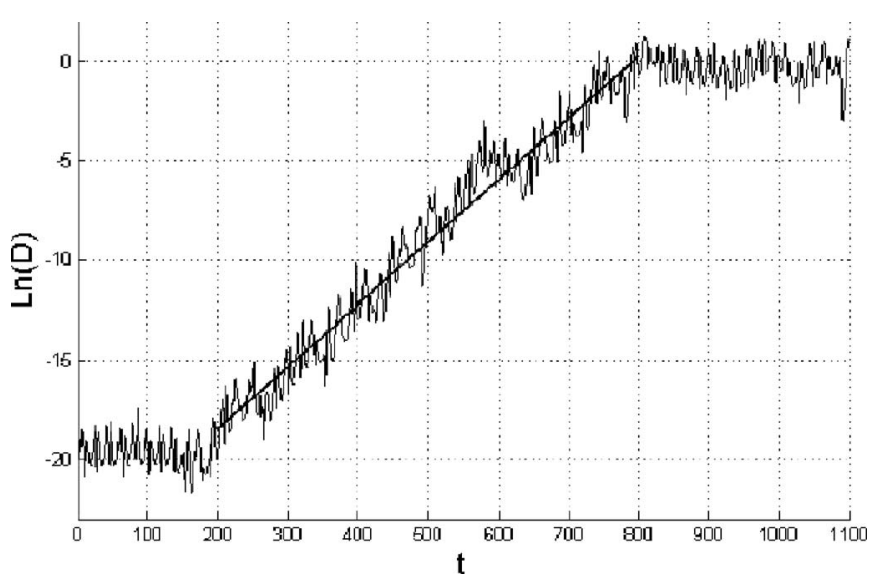

FIG. 9. Distance $D(t)$ between phase-space points initially close in the chaotic attractor at $\hat{\nu}=0.95$ in Fig. 8.

$$
\lambda_{4} \approx-0.95 \text {. }
$$

Their sum is in good agreement with the flow divergence as given by Eq. (4) with the normalized time in (5b),

$$
\sum_{1}^{4} \lambda_{i}=-1.78 \approx-2 \times(1-\hat{\Gamma})=-1.8 .
$$

Figure 9 shows the time variation of distance $D$ between initially close points in that chaotic attractor. We find $d \ln D / d t \approx 0.031$, which again is in good agreement with the value of $\lambda_{1}$.

Within the domain at low $\kappa$, to the left, where $P$ is unstable in Fig. 4, attractor bifurcation for $\hat{\Gamma}=0.1$ is typically much simpler. At $\kappa=0.1$, there is a single Hopf bifurcation near the $\hat{\nu}=\sqrt{0.17}$ limit value where $P$ becomes $P^{*}$. This is a subcritical bifurcation, the periodic orbit born there being thus unstable. That branch of unstable periodic orbits ends on a homoclinic orbit at $P^{*}$.

\section{RESISTIVE DAMPING MODEL}

In this section, we consider the resistive law, $\delta=\kappa^{2}$, characteristic of damping regimes in the lower right half of Fig. 2. Equation (9b) now reads

$$
\begin{aligned}
& \hat{\nu} \sin \beta=F_{R}(\kappa, \hat{\Gamma})-(1-2 \hat{\Gamma}) \cos \beta, \\
& F_{R} \equiv \frac{\sqrt{\kappa}}{1+\kappa}\left[\frac{(1+\kappa)^{2}}{1+\kappa^{2}}-2 \hat{\Gamma}\right] .
\end{aligned}
$$

With $F_{R}$ now depending of both $\hat{\Gamma}$ and $\kappa$, discussing the existence of $P$ must involve this last parameter.

Considering, first, values $\hat{\Gamma}<1 / 2$, we have $F_{R}>0$ throughout the range $0<\kappa<1$. For $\hat{\nu}<0$, condition (13a), then applying, can be rewritten here as

$$
F_{R}(\kappa, \hat{\Gamma})<1-2 \hat{\Gamma},
$$

which finally leads to 


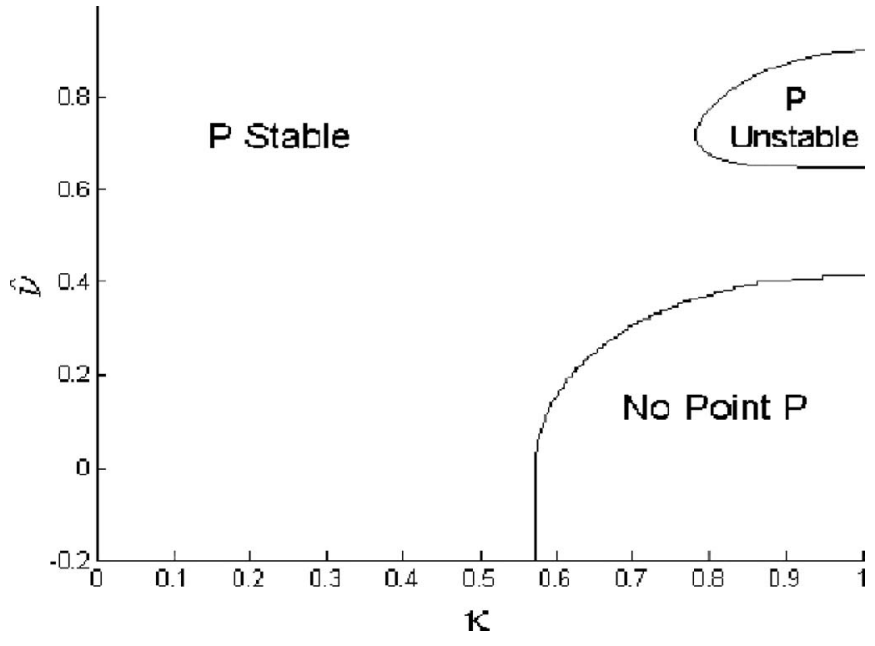

FIG. 10. Existence and stability of point $P$ at $\hat{\Gamma}=0.1$, in the resistive model.

$$
G^{-}(\kappa) \equiv \frac{2 \kappa \sqrt{\kappa}}{\left(1+\kappa^{2}\right)(1+\kappa-\sqrt{\kappa})}<1-2 \hat{\Gamma}
$$

or

$$
\begin{aligned}
& \kappa<\kappa_{\max }(\hat{\Gamma}), \\
& G^{-}\left(\kappa_{\max }\right)=1-2 \hat{\Gamma} .
\end{aligned}
$$

For positive $\hat{\nu}$, condition (12a) is clearly satisfied in the $\kappa$ range given by (30a). Point $P$ will thus exist for both polarizations, whatever the $|\hat{\nu}|$ value, in case $\kappa<\kappa_{\max }(\hat{\Gamma})$. We have $\kappa_{\max }$ moving from 0 to 1 as $\hat{\Gamma}$ decreases from $1 / 2$ to 0 .

For $\kappa>\kappa_{\max }(\hat{\Gamma})$, only LH polarization is possible, with condition $(12 \mathrm{a})$ reading

$$
\hat{\nu}>\hat{\nu}_{\min }(\hat{\Gamma}, \kappa) \equiv \sqrt{F_{R}^{2}-(1-2 \hat{\Gamma})^{2}} .
$$

As $\kappa$ increases from $\kappa_{\max }(\hat{\Gamma})$ to $1, \hat{\nu}_{\min }$ increases from 0 to

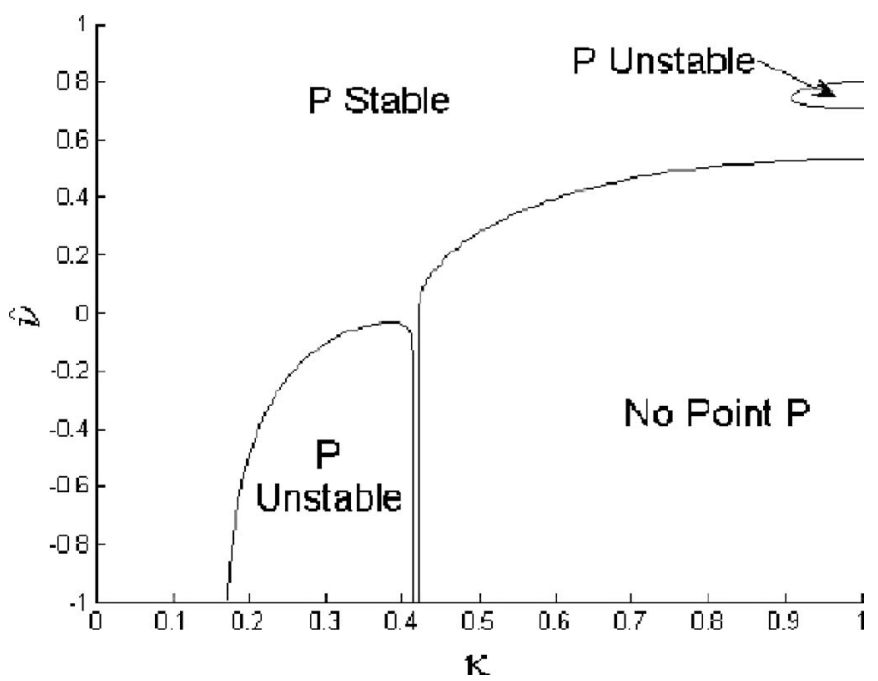

FIG. 11. Same as in Fig. 10 at $\hat{\Gamma}=0.2$.

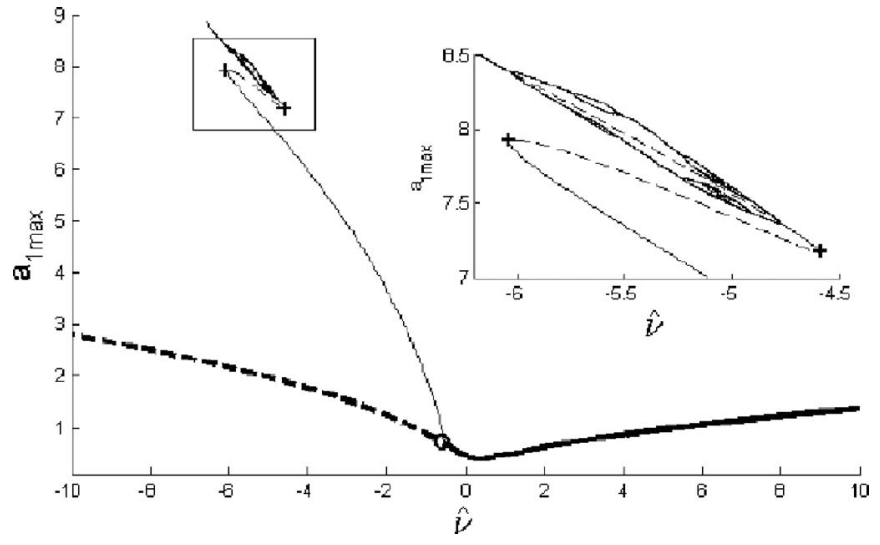

FIG. 12. Bifurcation diagram at $\hat{\Gamma}=0.2$ and $\kappa=0.195$ in the resistive model

$$
\hat{\nu}_{\min }(\hat{\Gamma}, 1) \equiv \sqrt{\hat{\Gamma}(2-3 \hat{\Gamma})}
$$

Note that as $\hat{\Gamma}$ decreases monotonously from $1 / 2, \hat{\nu}_{\min }(\hat{\Gamma}, 1)$ first increases from $1 / 2$ to a maximum $1 / \sqrt{ } 3$ at $\hat{\Gamma}=1 / 3$, $\kappa_{\max } \approx 0.26$, before dropping to zero at $\hat{\Gamma}=0$.

Figures 10 and 11 present both the existence and stability domains for $P$ at $\hat{\Gamma}=0.1$ and 0.2 , respectively, showing how $\kappa_{\max }$ moves to the left and $\hat{\nu}_{\min }$ at $\kappa=1$ increases, as $\hat{\Gamma}$ increases at low values. At $\hat{\Gamma}=0.2, P$ has become unstable in a $\kappa$ range to the left of $\kappa_{\max }$ at $\mathrm{RH}$ polarization, whereas the unstable $P$ domain at high $\kappa$ and LH polarization has shrunk. At $\hat{\Gamma}=0.5$, where there is no $P$ at negative $\hat{\nu}(\mathrm{RH}$ polarization), $P$ is unstable for $\kappa$ greater than about $2 / 3$ and $\hat{\nu}$ extending from large values down to $\hat{\nu}_{\text {min }}$.

At $\kappa=0.195$ in Fig. 11, in the domain to the left, at RH polarization and low $\kappa, P$ is unstable from about $\hat{\nu} \approx-0.7$ down. It loses stability through a supercritical Hopf bifurcation, the resulting periodic orbit losing stability itself through a cyclic fold at $\hat{\nu} \approx-6.1$ (Fig. 12). The unstable periodic orbit joins a stable one at a second cyclic fold; a chaotic attractor lies around $\hat{\nu} \approx-5.5$, between two period-doubling sequences. Figure 13 shows phase-space projections onto the planes $a_{1}-a_{2}, \beta-r$, of a chaotic attractor born from a loss of stability of $P$ in the small domain to the right in Fig. 11, at LH polarization and high $\kappa$.

For $\hat{\Gamma}>1 / 2$, only the case $\hat{\nu} F_{R}>0$, under condition (12b), may apply,

$$
\hat{\nu}^{2}>F_{R}^{2}-(1-2 \hat{\Gamma})^{2}>0 .
$$

Now $F_{R}$ changes sign at $\kappa_{0}(\hat{\Gamma})$ given by

$$
\left(1+\kappa_{0}\right)^{2}=2 \hat{\Gamma}\left(1+\kappa_{0}^{2}\right) .
$$

For $\kappa<\kappa_{0}(\hat{\Gamma})$, we have $F_{R}<0$, requiring $\hat{\nu}<0$, conditions (33) yielding

$$
-F_{R}(\kappa, \hat{\Gamma})>2 \hat{\Gamma}-1
$$

which one can readily verify it can be satisfied at no $\kappa$. No $\mathrm{RH}$ polarization is possible at $\hat{\Gamma}>1 / 2$. 


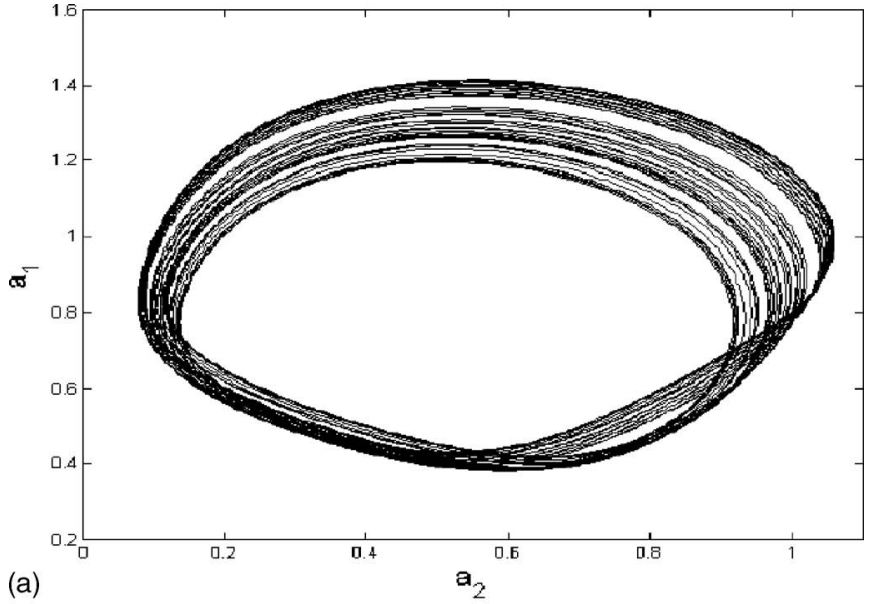

(a)

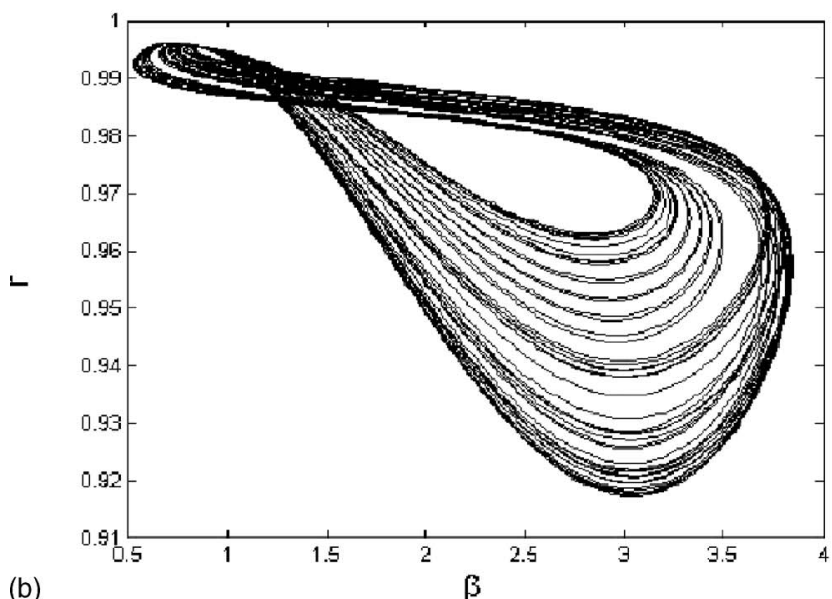

(b)

FIG. 13. Phase-space structure of chaotic attractor at $\hat{\Gamma}=0.2, \kappa=0.99$, and $\hat{\nu}=1.0$, in the resistive model; (a) projection in plane $a_{1}-a_{2}$, (b) projection in plane $\beta-r$.

For $\hat{\Gamma}>1 / 2$ and $\kappa>\kappa_{0}(\hat{\Gamma})$, we have $F_{R}>0$, requiring $\hat{\nu}>0$; we must have in turn, $F_{R}(\kappa, \hat{\Gamma})>2 \hat{\Gamma}-1$, which reads

$$
G^{+}(\kappa) \equiv \frac{2 \kappa \sqrt{\kappa}}{\left(1+\kappa^{2}\right)(1+\kappa+\sqrt{\kappa})}>2 \hat{\Gamma}-1
$$

or

$$
\begin{gathered}
\kappa>\kappa_{\min }(\hat{\Gamma})>\kappa_{0}(\hat{\Gamma}), \\
G^{+}\left(\kappa_{\min }\right)=2 \hat{\Gamma}-1 .
\end{gathered}
$$

Note that both $\kappa_{0}$ and $\kappa_{\min }$ collapse to 0 as $\hat{\Gamma} \rightarrow 1 / 2$. Throughout the range $\kappa>\kappa_{\min }$, we have

$$
\hat{\nu}>\hat{\nu}_{\min }(\hat{\Gamma}, \kappa) \equiv \sqrt{F_{R}^{2}-(1-2 \hat{\Gamma})^{2}} .
$$

As $\hat{\Gamma} \rightarrow 2 / 3$, we have $\kappa_{\min } \rightarrow 1$ (and $\kappa_{0} \rightarrow 3-\sqrt{ } 8$ ). There thus exists no point $P$ for $\hat{\Gamma}>2 / 3$.

\section{CONCLUSIONS}

We truncated the DNLS equation to three modes under resonant interaction (WRI), with waves 2 and 3 damped and wave 1 having a growth rate $\Gamma$. We took the wave-vector ratio $\kappa \equiv k_{2} / k_{3}<1$ and assumed the damping-rate ratio $\delta \equiv \gamma_{2} / \gamma_{3}<1$, too. We also took $\hat{\Gamma} \equiv \Gamma /\left(\gamma_{2}+\gamma_{3}\right)<1$, for the flow in the $4 \mathrm{D}$ phase space of three wave amplitudes and one relative phase to have negative divergence. A fourth dimensionless parameter is a normalized frequency mismatch, $\hat{\nu} \equiv \nu /\left(\gamma_{2}+\gamma_{3}\right)$, with $\nu$ given by Eq. (3), and $\nu>0(<0)$ for $\mathrm{LH}(\mathrm{RH})$ polarization. We have discussed how gross features in the dynamical behavior are affected by both the damping model and wave polarization.

The 4D flow may present up to two fixed points. One is always unstable. If the other fixed point $(P)$ exists, the loss of its stability gives rise to extremely complex dynamics. We found parametric domains exhibiting stable and unstable periodic orbits, Hopf supercritical bifurcations, two cyclic folds, three Feigenbaum sequences ending in chaotic attractors, two blue sky catastrophes (boundary crisis), and one explosive (Shilnikov-like) bifurcation leading to intermittency I-type chaos. Up to five attractors may coexist at a parametric point.

Point $P$ does not exist for $2 / 3<\hat{\Gamma}<1$ independently of model. For Landau-type damping $(\gamma \propto k \rightarrow \delta=\kappa), P$ does not exist either at $\mathrm{RH}$ polarization; it exists at $\mathrm{LH}$ polarization for $\hat{\nu}>\sqrt{\hat{\Gamma}(2-3 \hat{\Gamma})}$. There are two $\hat{\nu}-\kappa$ instability domains, with more complex dynamics at the high $\hat{\nu}$, high $\kappa$ end. Landau damping is singular in the sense that other damping models do allow for $\mathrm{RH}$ polarization.

For resistive damping $\left(\gamma \propto k^{2} \rightarrow \delta=\kappa^{2}\right) P$ exists at RH polarization for $\hat{\Gamma}<1 / 2$ and $\kappa$ less than some maximum $\kappa_{\max }(\hat{\Gamma})$, with $P$ unstable for not too small $\hat{\Gamma}$ or $\kappa$. Point $P$ exists at LH polarization, with $\hat{\Gamma}<1 / 2$, for all $\kappa$; with $\hat{\nu}$ greater than some $\hat{\nu}_{\text {min }}(\hat{\Gamma}, \kappa)$ in case $\kappa>\kappa_{\max }(\hat{\Gamma})$; it exists in the range $1 / 2<\hat{\Gamma}<2 / 3$, for $\kappa$ greater than some minimum $\kappa_{\text {min }}(\hat{\Gamma})$, with $\hat{\nu}$ again greater than some $\hat{\nu}_{\text {min }}(\hat{\Gamma}, \kappa)$. At this high $\hat{\Gamma}$ regime, $P$ is unstable over most of its $\kappa-\hat{\nu}$ domain of existence, independently of damping model.

\section{ACKNOWLEDGMENTS}

This work was supported by the Spanish Ministry of Science and Technology under Grant No. ESP2004-01511. Work by S.E. was supported by PIP 5692 of CONICET and PICTO-UNRC-2005 No. 30339.

\footnotetext{
${ }^{1}$ A. Rogister, Phys. Fluids 14, 2733 (1971); K. Mio, T. Ogino, K. Minami, and S. Takeda, J. Phys. Soc. Jpn. 41, 265 (1976).

${ }^{2}$ E. Mjolhus, J. Plasma Phys. 19, 437 (1978); Phys. Scr., T T122, 135 (2006).

${ }^{3}$ C. F. Kennel, B. Buti, T. Hada, and R. Pellat, Phys. Fluids 31, 1949 (1988); T. Hada, C. F. Kennel, and B. Buti, J. Geophys. Res. 94, 65 (1989).

${ }^{4}$ D. J. Kaup and A. C. Newell, J. Math. Phys. 19, 798 (1978); X-J. Chen and W. K. Lam, Phys. Rev. E 69, 066604 (2004).

${ }^{5}$ K. Stasiewicz, P. K. Shukla, G. Gustaffson, S. Buchert, B. Lavraud, B. Thide, and Z. Klos, Phys. Rev. Lett. 90, 085002 (2003); K. Stasiewicz, ibid. 93, 125004 (2004).

${ }^{6}$ S. R. Spangler, in Nonlinear Waves and Chaos in Space Plasmas, edited by T. Hada and H. Matsumoto (Terrapub, Tokyo, 1997), pp. 171-224.

${ }^{7}$ S. R. Spangler, Phys. Fluids 29, 2535 (1986); Y. Nariyuki and T. Hada, Earth, Planets Space 57, e9 (2005).

${ }^{8}$ S. Ghosh and K. Papadopoulos, Phys. Fluids 30, 1371 (1987).
} 
${ }^{9}$ T. Hada, C. F. Kennel, B. Buti, and E. Mjolhus, Phys. Fluids B 2, 2581 (1990); A. C. L. Chian, F. A. Borotto, and W. D. Gonzalez, Astrophys. J. 505, 993 (1998); F. A. Borotto, A. C. L. Chian, T. Hada, and E. L. Rempel, Physica D 194, 275 (2004); E. L. Rempel and A. C. L. Chian, J. Atmos. Sol.-Terr. Phys. 67, 1837 (2005).

${ }^{10}$ E. Marsch and S. Liu, Ann. Geophys. 11, 227 (1993); R. Bruno, V. Carbone, P. Veltri, E. Pietropaolo, and B. Bavassano, Planet. Space Sci. 49, 1201 (2001)

${ }^{11}$ J. R. Sanmartin, O. Lopez-Rebollal, E. del Rio, and S. Elaskar, Phys. Plasmas 11, 2026 (2004).

${ }^{12}$ R. A. Miranda, E. L. Rempel, A. C. L. Chian, and F. A. Borotto, J. Atmos. Sol.-Terr. Phys. 67, 1852 (2005).

${ }^{13}$ S. D. Drell, H. M. Foley, and M. A. Ruderman, J. Geophys. Res. 70, 3131
(1965); A. Barnett and S. Olbert, ibid. 91, 10117 (1986); J. R. Sanmartín and M. Martínez-Sanchez, ibid. 100, 1677 (1995).

${ }^{14}$ J. R. Sanmartin and R. D. Estes, J. Geophys. Res. 102, 14625 (1997).

${ }^{15}$ G. V. Khazanov, N. H. Stone, E. N. Krivorutsky, and E. W. Liemohn, J. Geophys. Res. 105, 15835 (2000); G. V. Khazanov, N. H. Stone, E. N. Krivorutsky, K. V. Gamayunov, and E. W. Liemohn, ibid. 106, 10565 (2001); J. R. Sanmartin and R. D. Estes, ibid. 107, SIA 2-1 (2002).

${ }^{16}$ G. Vahala and D. Montgomery, Phys. Fluids 14, 1137 (1971); M. P. Hertzberg, N. F. Cramer, and S. V. Vladimirov, Phys. Plasmas 10, 3160 (2003).

${ }^{17}$ N. F. Cramer, The Physics of Alfiven Waves (Wiley-VCM, Berlin, 2001), Sec. 5.5 .

${ }^{18}$ E. Mjolhus, J. Plasma Phys. 16, 321 (1976). 
Physics of Plasmas is copyrighted by the American Institute of Physics (AIP). Redistribution of journal material is subject to the AIP online journal license and/or AIP copyright. For more information, see http://ojps.aip.org/pop/popcr.jsp 